disease, 2 were premature and 2 had hypoparathyroidism. None had nutritional abnormalities. Five died and 2 had neurologic abnormalities at follow-up. Prognosis was related more to associated medical conditions than the seizures. (Lynch BJ, Rust RS. Natural history and outcome of neonatal hypocalcemic and hypomagnesemic seizures. Pediatr Neurol July 1994;11:2327). (Respond: Dr Rust, Dept Neurology, University of Wisconsin-Madison Medical School, H6/571 Clinical Science Center, 600 Highland Ave, Madison, WI 53792).

COMMENT. The associated congenital heart disease in almost $50 \%$ of these patients is remarkable and indicates the need for careful monitoring of serum calcium and magnesium levels before and after open heart surgery. Although hypocalcemic seizures caused by high phosphate content of cow's milk-based infant formula is now a rare event, seizures related to low calcium and magnesium may be experienced in infants with parathyroid disorders, low birth weight, prematurity, fatty diarrhea, gastrointestinal disease, chronic nephropathy, and cardiac disease, in addition to infants with asphyxia, brain hemorrhage, and other cerebral lesions. In four infants with hypocalcemia treated personally at the Mayo Clinic, a history of cerebral anoxia or intracranial hemorrhage was reported at birth, and in one infant, an intracranial tumor was diagnosed. Seizures did not respond to intravenous calcium gluconate and a neurogenic hypocalcemia was postulated. (Millichap JG. Nutrition, Diet, and Child's Behavior. Springfield, CC Thomas, 1986).

\title{
PROLONGED QT SYNDROME PRESENTING AS EPILEPSY
}

Two patients, ages 19 and 12 years, and a review of 8 previous cases of prolonged QT syndrome presenting as epilepsy are reported from the Department of Neurology and Hospital for Joint Diseases Epilepsy Center, New York University, New York. The 19 year old woman presented with "blackout spells" at 13 years. Some were a transient light-headedness and weakness, while other attacks progressed to tonic-clonic seizures with loss of consciousness. CT, EEG, ECG, and Holter monitor were normal. Echocardiogram showed mitral valve prolapse. Carbamazepine was ineffective, and within 2 months she was found dead at home. Review of ECGs revealed a prolonged QT interval, previously unrecognized. The 12 year old boy was referred with intractable seizures since 4 years of age. He also had "panic attacks" with tachycardia, dyspnea, palm sweating, and limpness. Numerous AEDs were without benefit. EEG and ECG were normal. A Holter monitor revealed ventricular tachycardia and prolonged QT syndrome. Of a total of 10 patients, 5 had family histories consistent with congenital prolonged QT syndrome (ie. sudden death or deafness). The first convulsion occurred at an average age of 4 years. Time to diagnosis ranged from 1 to 28 years. Presyncope and "lifelessness" prior to seizures were common complaints. The beta blocker, propranolol, the mainstay of therapy, is successful in most patients. Some require a pacemaker or implantable cardiac defibrillator. (Pacia SV et al. The prolonged QT syndrome presenting as epilepsy: A report of two cases and literature review. Neurology Aug 1994;44:1408-1410). (Reprints: Dr Steven Pacia, Dept of Neurology, Hospital for Joint Diseases, 301 East 17 th St, New York, NY 10003).

COMMENT. Diagnosis of this life-threatening condition may be difficult, but several factors should alert the neurologist to the cardiac origin of the seizures: 1) history of tachycardia, and presyncopal or lifeless 
feelings prior to onset of convulsive attacks; 2) normal neurologic exam and EEG: 3) family history of deafness, cardiac arrhythmia, or unexpected sudden death; and 4) lack of response to antiepileptic drugs. Familial, autosomal recessive or dominant, and acquired types of prolonged QT interval are recognized. The mortality may be as high as $70 \%$ if unrecognized and untreated.

\section{VIDEO GAME-INDUCED SEIZURES}

Fifteen patients, ages 9 to 15 years, who experienced epileptic seizures while playing video games are reported from St Thomas's Hospital, London, UK. An additional 20 patients in 12 reports in the literature are reviewed, and 3 further patients are described in an addendum. The majority had the first seizure as a result of the video game. Seizure patterns were generalized tonic clonic in two thirds; some had absence and $30 \%$ had juvenile myoclonic epilepsy. Photosensitivity occurred in $70 \%$, while excitement, fatigue, sleep deprivation, and cognitive processing were important precipitants in others. Partial, mainly occipital, seizures occurred in 29\%. Management was individualized, and AEDs were not always necessary. (Ferrie CD et al. Video game induced seizures. I Neurol Neurosurg Psychiatry Aug 1994;57:925-931). (Respond: Dr CD Ferrie, Dept Clinical Neurophysiology and Epilepsy, St Thomas's Hospital, London SE1 7EH, UK).

COMMENT. Video game seizures are reflex epilepsies, generalized or partial, and a feature of various idiopathic epileptic syndromes. Both photic and non-photic precipitants are involved. The avoidance of the precipitant may prevent the progression of minor absences, jerks, or visual phenomena to a generalized tonic clonic epilepsy.

See Ped Neur Briefs April 1994, p 28, for a previous report of 10 patients seen at the University of Washington, Seattle, and a review of 20 cases cited in the literature. The comment that video game related seizures are more common than previously recognized appears to be confirmed.

\section{ANTIEPILEPTIC DRUGS}

\section{CEREBELLAR ATROPHY WITH PHENYTOIN AND EPILEPSY}

Cerebellar size measured by MRI was studied in a group of 36 adults (21 to 54 years, mean age 34 years) with intractable partial epilepsy treated with phenytoin longer than 4 years at the Epilepsy Center of the Long Island Jewish Medical Center, New Hyde Park, NY. Patients with IQ $<70$, ethanol abuse, status epilepticus, and neurodegenerative disorders were excluded. Measurements were compared to a group of control patients examined because of headache or dizziness. Mean duration of phenytoin exposure was 14 years (range, 4 to 30 years). Mean maximum dosage was $450 \mathrm{mg}$ daily (range, 300 to $700 \mathrm{mg}$ ). All patients had received various AEDs other than phenytoin. Moderate to severe cerebellar atrophy was found in $9(25 \%)$ patients and mild atrophy in 12 (33\%). The MRI was normal in 15 (42\%) phenytoin exposed patients and in 33 (94\%) controls. A correlation between cerebellar atrophy ratings and variables reflective of seizure severity or degree of phenytoin exposure could not be demonstrated. (Ney GC et al. Cerebellar atrophy in patients with long-term phenytoin exposure and epilepsy. Arch Neurol Aug 